\title{
Bioqualité Des Formes De Dissémination Des Protozoaires Flagellés Entériques Dans Les Eaux Souterraines (Sources Et Puits) En Zone Anthropisée (Yaoundé-Cameroun)
}

\section{Ajeagah Gideon Aghaindum Asi Quiggle Atud Nola Moïse}

Laboratoire d'Hydrobiologie et Environnement, Faculté des Sciences, Université de Yaoundé I, Yaoundé, Cameroun

doi: 10.19044/esj.2016.v12n32p554 URL:http://dx.doi.org/10.19044/esj.2016.v12n32p554

\begin{abstract}
With an aim of characterizing the cysts of flagellated enteric protozoa present in groundwater of the town of Yaoundé, a descriptive and analytical study was carried out from February to July 2015 on the samples of 6 springs and 6 wells in 6 quarters of the city. The physico-chemical analysis proceeded at the same time on the field and at the laboratory. The observation of the cysts of the flagellated protozoa was done under the inverted microscope of mark Olympus CK2 to the objective 40X after concentration of the samples according to the method of sedimentation and the method of floating. The physicochemical analysis revealed slightly acid water (6, $04 \pm 0,49$ U.C), average mineral-bearing (533, $70 \pm 346,18$ $\mu \mathrm{S} / \mathrm{cm})$ and rich in suspended matter (14, $14 \pm 11,35 \mathrm{mg} / \mathrm{L})$. The biological analyses revealed the presence of the cysts of the flagellated Protozoa with average densities of $162 \pm 115$ cysts/L for Giardia intestinalis, $262 \pm 280$ cysts /L for Chilomastix mesnili, 89 \pm 101 cysts /L for Enteromonas hominis and $72 \pm 80$ cysts /L for Retortamonas intestinalis. In general, the greatest densities of cysts were recorded during the rainy season. The statistical tests revealed positive correlation between the cystic densities with MES, the color, turbidity and the organic matter $(\mathrm{p}<0,05)$, and also negative correlation with nitrate $(\mathrm{p}<0,05)$.This very high parasitological pollution makes water unfit for consumption. The use of untreated spring waters and wells could constitute a significant heath for the households living in the zone of study.
\end{abstract}


Keywords: Cysts, enteric flagellated protozoa, groundwater, microbiological contamination, physico-chemistry

\section{Resume}

Dans le but de caractériser les kystes des protozoaires flagellés entériques présents dans les eaux souterraines de la ville de Yaoundé, une étude descriptive et analytique a été menée sur un échantillon de six (6) sources et six (6) puits dans six (6) quartiers de la ville, de février à juillet 2015. Les analyses physico-chimiques se sont déroulées à la fois sur le terrain et au laboratoire d'Hydrobiologie et Environnement de l'Université de Yaoundé I. L’observation des kystes de protozoaires flagellés s'est faite au microscope inversé de marque Olympus CK2 à l'objectif 40X après concentration des échantillons suivant les méthodes de sédimentation et de flottation. Les analyses physico-chimiques ont révélé des eaux légèrement acides (6,04 $\pm 0,49$ U.C), moyennement minéralisées (533,70 \pm 346,18 $\mu \mathrm{S} / \mathrm{cm})$ et riches en matières en suspension $(14,14 \pm 11,35 \mathrm{mg} / \mathrm{L})$. Les analyses biologiques ont révélé la présence des kystes de protozoaires

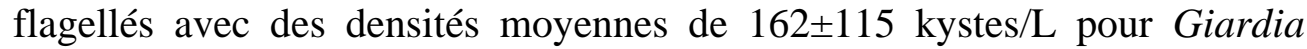
intestinalis, $262 \pm 280$ kystes/L pour Chilomastix mesnili, 89 \pm 101 kystes/L pour Enteromonas hominis et $72 \pm 80$ Kystes/L pour Retortamonas intestinalis. Dans l'ensemble, les densités de kystes ont été plus elevées pendant la petite saison de pluies. La dynamique d'abondance de ces entéroprotozoaires flagellés est significativement et positivement corrélée aux MES, à la couleur, à la turbidité et aux matières organiques de l'eau ( $p$ $<0,05)$, d'une part et négativement à la teneur en nitrates $(\mathrm{p}<0,01)$, d'autre part. Cette pollution parasitologique très élevée rend l'eau impropre à la consommation. L'utilisation des eaux de source et de puits non traitées constituerait un risque sanitaire.

Mots clés: Eaux souterraines, contamination microbiologique, kystes, protozoaires flagellés entériques, physico-chimie

\section{Introduction}

L'eau, indispensable à la vie, est le premier élément constitutif des êtres vivants et de leur environnement. Toutefois, depuis la fin du XIX ${ }^{\text {ième }}$ siècle, l'eau est reconnue comme un moyen de contamination de l'homme (Bertrand et al., 2005). La mise en évidence de l'infestation par voie hydrique des nombreux organismes pathogènes a conduit au développement des techniques de traitement de l'eau de boisson. L'accès à l'eau potable reste inégal entre les pays du monde et particulièrement en Afrique subsaharienne (Bertrand et al., 2005). L’Organisation Mondiale de la Santé 
(OMS, 2011) estime à 1,1 milliards le nombre de personnes qui n’y ont pas accès.

Au Cameroun, 65\% de la population n'a pas accès à l'eau courante (Camwater, 2011). Seul 50\% des besoins en eau sont couvertes pour les populations de la capitale politique (OMS, 2012).Cette insuffisance couplée aux difficultés financières obligent de nombreux ménages à s’alimenter dans les eaux souterraines en occurrence les puits (35.9\%) et sources (5.6 \%) de la ville (Institut National de la Statistique, 2013), sans se préoccuper bien souvent de la qualité (Nola et al., 1998).

Les eaux souterraines en milieu urbain, subissent pourtant de multiples contraintes dues à l'absence des systèmes d'assainissement et une croissance démographique galopante (Foster, 2001 ; Nkhuwa, 2003). Ceci se traduit très souvent par la production et le rejet considérables de déchets dans la nature, lesquels sont susceptibles de contaminer la nappe phréatique (Dejoux ,1988; Nkhuwa, 2003). En effet, certaines études ont révélé la présence des bactéries et agents chimiques dans les eaux souterraines à Meknès au Maroc (Belghiti et al,. 2013) et à Yaoundé au Cameroun (Nola et al.,1998 ) démontrant la contamination de la nappe. D’autres études ont montré la présence des kystes de protozoaires flagellés intestinaux (Giardia) dans les eaux souterraines au Benin (Degbey et al., 2010) et à Ngaoundéré Mbawala et al., 2010). Mais, aucune attention n’a encore été accordée à la contamination des eaux souterraines par les formes de résistance de protozoaires flagellés à Yaoundé. Les travaux menés à Yaoundé sur ce groupe d'organismes par Ajeagah et al. (2010) ont montré leur présence dans certains cours d'eau du réseau hydrographique du Mfoundi. Leur transmission se fait par voie orale et bon nombre d'épidémies ont été associées à leur présence dans les milieux aquatiques (Yousefi et al., 2009).

Les effets induits par un manque d'eau ou par une eau de mauvaise qualité, sont souvent néfastes et causent chaque année des millions de morts dans le monde entier. Selon l'OMS (2003), 80\% de maladies dans les pays en développement sont liées à l'eau. Parmi ces maladies, on peut citer l'amibiase, la cryptosporidiose, le choléra, la giardiose entre autres. Elles sont causées par divers groupes d’organismes (virus, bactéries, protozoaires etc...). De tous ces organismes, les protozoaires flagellés intestinaux sont classés au premier rang de parasitose enteropathogène (Aubry et al., 1986). En effet, contrairement à de nombreux pathogènes dont la dose infectieuse est très élevée, l'ingestion de 10 kystes de flagellés pathogènes suffit à infecter l'homme (Wolfe, 1992; Johan, 2010).

Dans l'environnement, ces organismes sont retrouvés sous forme de kystes qui assurent leur dissémination (Petithory et al., 1998). De toutes ces formes kystiques, le kyste de Giardia intestinalis est le plus virulent (Lacoste, 2009). Il est responsable de la giardiose qui se manifeste par des 
diarrhées aiguës, des douleurs abdominales, des nausées et des pertes de poids (Raccurt et al., 2006) pouvant particulièrement se compliquer en syndromes d'anémie et malabsorption ainsi que chez les personnes immunodéprimées et non traitées (Euzeby, 2002; Bertrand et al., 2005). On estime l'incidence mondiale de la giardiose à 200 millions de cas par année (OMS, 1994).

Vu la menace permanente de ces groupes d'organismes sur la santé, une étude a été menée à Yaoundé sur la caractérisation des kystes de protozoaires flagellés présents dans quelques sources et puits de la ville.

\section{Materiel et methodes \\ Materiel \\ Présentation de la zone d'étude}

La ville de Yaoundé, capitale politique du Cameroun, est située au Sud de la région du Centre entre $3^{\circ} 30^{\prime}$ et $3^{\circ} 58^{\prime}$ de latitude Nord et entre $11^{\circ} 20^{\prime}$ et $11^{\circ} 40^{\prime}$ de longitude Est (Suchel, 1972). Et est située à près de 750 m d'altitude, elle est caractérisée par un climat particulier à 4 saisons dit « climat Yaoundéen » (Suchel, 1972) comprenant : une grande saison sèche (GSS) qui s'étend de mi-novembre à mi-mars, une petite saison de pluies (PSP) qui va de mi-mars à fin mai, une petite saison sèche (PSS) de juin à août, une grande saison de pluies (GSP) qui va de septembre à mi-novembre. Le régime thermique est chaud et varie très peu. Ainsi, les températures moyennes mensuelles oscillent entre $22,4^{\circ} \mathrm{C}$ et $27,2^{\circ} \mathrm{C}$. La pluviométrie moyenne annuelle est de $1576 \mathrm{~mm}$

La roche-mère, qui constitue le substratum géologique des sols de Yaoundé, dérive d'un matériau quartzo-feldspathique plus ou moins micacé (Pelletier, 1969), d'où la forte acidité de ses sols avec un pH de 4,5 à 5,5 dans les couches superficielles. La végétation est de type forêt dense humide semi-décidue et le réseau hydrographique est principalement constitué par le Mfoundi et ses affluents.

\section{Cadre d'étude}

L'étude s'est déroulée en deux phases. La première phase est la prospection allant de novembre 2014 à janvier 2015. Les multiples descentes sur le terrain, ont permis de choisir les stations d'échantillonnage en fonction du niveau piézomètre, des proximités des sources de pollution, l'accord du propriétaire et fréquentation de l'ouvrage. Douze stations d'échantillonnage ont été choisies, dont 6 sources (MoS, NkS, MeS, MlS, EtS et ObS) et 6 puits (MoP, NkP, MeP, MlP, EtP et ObP) repartis dans les quartiers de Mokolo, Nkomkana, Melen I, Melen VII, Obili et Etoug-Ebé (Figure1). La deuxième phase est l'échantillonnage, qui s'est étendue du mois de février 
2015 à juillet 2015, soit six (6) mois avec une fréquence d'un prélèvement par mois.

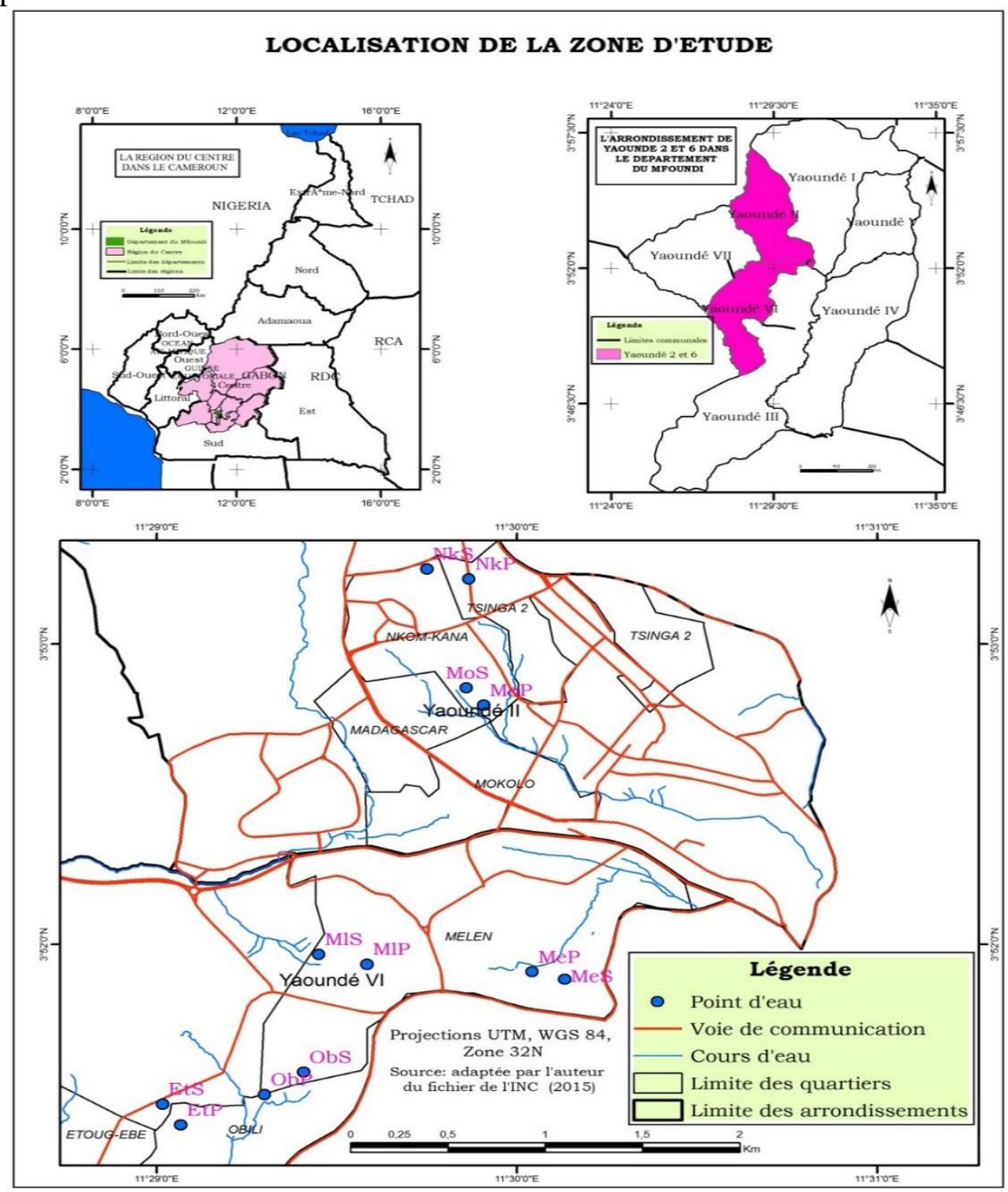

Figure 1: Carte de localisation des stations d'échantillonnages (source: Institut National de Cartographie 2015 modifiée).

\section{Coordonnées géologiques, description des stations d'étude et les usages (Tableau 1)}

Dans l'ensemble, les eaux des sources et des puits sont utilisées pour la boisson, la baignade, brossage des dents, la cuisson des repas, lessive, l'arrosage des cultures et les activités ménagères. Dans certains cas, la puisette ou seau est privée et déposée au sol après puisage. 


\begin{tabular}{|c|c|c|c|c|c|c|}
\hline $\begin{array}{c}\text { Points de } \\
\text { prélèvement }\end{array}$ & $\begin{array}{l}\text { Coordonnées } \\
\text { géographiques }\end{array}$ & $\begin{array}{l}\text { Altitudes } \\
\text { (m) }\end{array}$ & $\begin{array}{c}\text { Distance } \\
\text { aux } \\
\text { toilettes } \\
\text { (m) }\end{array}$ & $\begin{array}{c}\begin{array}{c}\text { Distance } \\
\text { effluents }\end{array} \\
\text { domestiques } \\
\text { (m) }\end{array}$ & $\begin{array}{l}\text { Niveau de } \\
\text { protection }\end{array}$ & $\begin{array}{c}\text { Traitement } \\
\text { chimique }\end{array}$ \\
\hline MoS & $\begin{array}{l}03^{\circ} 52^{\prime} 48.5^{\prime \prime} \mathrm{N}, \\
011^{\circ} 29^{\prime} 52.3^{\prime \prime} \mathrm{E}\end{array}$ & 722 & 6 & 5 & $\begin{array}{c}\text { Non } \\
\text { aménagée }\end{array}$ & Oui \\
\hline MoP & $\begin{array}{l}03^{\circ} 52^{\prime} 46.7^{\prime}, \mathrm{N} \\
11^{\circ} 2953.8^{\prime \prime} \mathrm{E}\end{array}$ & 726 & 5 & 1 & $\begin{array}{c}\text { Margelle } \\
\text { sans } \\
\text { couvercle }\end{array}$ & Non \\
\hline NkS & $\begin{array}{l}03^{\circ} 53^{\prime} 15.5^{\prime \prime} \mathrm{N}, \\
011^{\circ} 29^{\prime} 45.3^{\prime \prime} \mathrm{E}\end{array}$ & 762 & 15 & 2,5 & aménagée & Non \\
\hline NkP & $\begin{array}{l}03^{\circ} 53^{\prime} 13.1^{\prime \prime} \mathrm{N}, \\
011^{\circ} 29 \text { ' 52.2'” }\end{array}$ & 756 & 5 & 1 & $\begin{array}{c}\text { Margelle } \\
\text { partielle } \\
\text { sans } \\
\text { couvercle }\end{array}$ & Non \\
\hline MeS & $\begin{array}{l}03^{\circ} 51^{\prime} 53.1^{\prime \prime}, \mathrm{N}, \\
011^{\circ} 30^{\prime} 08.1^{\prime \prime} \mathrm{E}\end{array}$ & 731 & 3,5 & 8 & $\begin{array}{l}\text { Non } \\
\text { aménagée }\end{array}$ & Oui \\
\hline MeP & $\begin{array}{l}03^{\circ} 51^{\prime} 52.9^{\prime \prime} \mathrm{N} \\
011^{\circ} 30^{\prime} 06.6 \text { ' } \mathrm{E}\end{array}$ & 733 & 8 & 0 & $\begin{array}{l}\text { Margelle } \\
\text { sans } \\
\text { couvercle }\end{array}$ & Oui \\
\hline MIS & $\begin{array}{l}03^{\circ} 51^{\prime} 58.2^{\prime} \text { 'N, } \\
011^{\circ} 29^{\prime} 27.4^{\prime \prime} \mathrm{E}\end{array}$ & 724 & 8 & 3 & $\begin{array}{l}\text { Non } \\
\text { aménagée }\end{array}$ & Non \\
\hline MIP & $\begin{array}{l}03^{\circ} 51^{\prime} 56.1^{\prime \prime} \mathrm{N} \\
011^{\circ} 29^{\prime} 35.9 \text { '’ }\end{array}$ & 747 & 3 & 0 & $\begin{array}{c}\text { Margelle } \\
\text { sans } \\
\text { couvercle }\end{array}$ & Oui \\
\hline EtS & $\begin{array}{l}03^{\circ} 51^{\prime} 27.7^{\prime \prime} \mathrm{N}, \\
011^{\circ} 29 \text {, } \\
\end{array}$ & 727 & 5 & 0 & $\begin{array}{c}\text { Non } \\
\text { aménagée }\end{array}$ & Non \\
\hline EtP & $\begin{array}{l}03^{\circ} 51^{\prime} 36.6^{\prime \prime} \mathrm{N}, \\
011^{\circ} 2907.7^{\prime \prime} \mathrm{E}\end{array}$ & 727 & 16 & 9 & $\begin{array}{c}\text { Margelle } \\
\text { partielle } \\
\text { sans } \\
\text { couvercle }\end{array}$ & Oui \\
\hline ObS & $\begin{array}{l}03^{\circ} 51^{\prime} 32.2^{\prime \prime} \mathrm{N}, \\
011^{\circ} 29^{\prime} 25.2^{\prime \prime} \mathrm{E}\end{array}$ & 721 & 5 & 0 & $\begin{array}{c}\text { Non } \\
\text { aménagée }\end{array}$ & Non \\
\hline ObP & $\begin{array}{l}03^{\circ} 51^{\prime} 30.7^{\prime} \text { 'N, } \\
011^{\circ} 29^{\prime} 18.8 \text { '’E }\end{array}$ & 715 & 5 & 3 & $\begin{array}{c}\text { Margelle } \\
\text { partielle } \\
\text { sans } \\
\text { couvercle }\end{array}$ & Oui \\
\hline
\end{tabular}

\section{Methodes}

\section{Mesures des paramètres morphométriques et hydrologiques}

La hauteur de la colonne d'eau, la profondeur et le diamètre du puits ont été mesurés à l'aide d'une corde lestée et graduée. Les coordonnées géographiques et l'altitude des différentes stations ont été obtenues à l'aide d'un GPS Harmin model Etrex 30. Le débit d'écoulement d'eau des sources a été déterminé par la formule $Q=V S$, avec $Q=$ débit $\mathrm{m}^{3} / \mathrm{s}, V=$ vitesse d'écoulement en $\mathrm{m} / \mathrm{s}, S$ =section d'eau en $\mathrm{m}^{2}$.

\section{Mesures des paramètres physico-chimiques}

Les analyses physico-chimiques se sont déroulées à la fois sur le terrain et au laboratoire suivant la méthode APHA (1998) et Rodier et al. (2009). Pour les paramètres mesurés au laboratoire, les échantillons d'eau ont été prélevés à l'aide des flacons en polyéthylène à double bouchage de 250 et $1000 \mathrm{~mL}$, et ramenés au laboratoire en enceinte réfrigérée. La température, teneur en oxygène, la conductivité électrique, les TDS et le $\mathrm{pH}$ 
ont été mesurés sur le terrain à l'aide d’un multiparamètre de marque HANNA HI 9829. Les MES, la turbidité, la couleur et la teneur de l'eau en chlore libre ont été mesurés au laboratoire à l'aide d'un spectrophotomètre $\mathrm{HACH} \mathrm{DR} / 3900$. Les mesures des teneurs de l'eau en nitrates et en orthophosphates ont été faites par colorimétrie au spectrophotomètre HACH $\mathrm{DR} / 3900$ alors que les mesure de la teneur de l'eau en $\mathrm{CO}_{2}$ dissous, l’alcalinité, l’oxydabilité a été réalisée par volumétrie.

\section{Etude de protozoaires flagellés}

Les échantillonnages d'eau pour l'identification des kystes de protozoaires flagellés ont été effectués dans les sources, les puits à des endroits caractérisés par une accumulation de la matière organique ou la présence de l'herbier. Les échantillons d'eau ainsi collectés ont été immédiatement introduits dans des flacons stériles en polyéthylène de 1000 $\mathrm{mL}$ puis transportés au laboratoire dans une glacière. Au laboratoire, les échantillons ont été placés pour décantation pendant 24 heures. Le surnageant a été versé et le culot restant a été mesuré à l'aide d'une éprouvette. Deux méthodes ont été utilisées pour les observations: la méthode de sédimentation simple et la méthode de flottation de Faust.

\section{Méthode de sédimentation simple}

Après homogénéisation du culot, $5 \mathrm{~mL}$ d’échantillon ont été prélevés à l'aide d'une seringue graduée et introduits dans un tube à essai. A cela, $1 \mathrm{~mL}$ de formol $10 \%$ a été ajouté pour assurer la fixation des organismes, $5 \mathrm{~mL}$ d'eau distillée et 2 gouttes de Lugol ont été successivement ajoutés. Le mélange obtenu a été porté à centrifugation à 500 tours/min pendant 5 minutes à l'aide d'une centrifugeuse de marque MEDIFRIGER. Par la suite, 1 à 2 goutes de culot ont été prélevées à l'aide d'une pipette et montées entre lame et lamelle.

\section{Méthode par flottation au sulfate de zinc ou technique de Faust (1938).}

Cette méthode permet la flottation des kystes. Après homogénéisation du culot, $5 \mathrm{~mL}$ de l'échantillon ont été prélevés à l'aide d'une seringue graduée et introduits dans un tube à essai. A cela, $1 \mathrm{~mL}$ de formol à $10 \%$ a été ajouté et $3 \mathrm{~mL}$ de sulfate de zinc à 33\% ont été successivement ajoutés. Le mélange obtenu a été porté à la centrifugation à 500 tours/min pendant 5 minutes à l'aide d'une centrifugeuse de marque MEDIFRIGER. Un volume donné du culot est prélevé à l'aide d'une pipette le surnageant dans lequel se trouve les parasites et une goûte est étalée sur lame porte-objet et recouverte d'une lamelle.

La préparation obtenue a été placée sur la platine du microscope inversé de marque Olympus CK2 pour l’observation des kystes après réglage 
au grossissement 40X. L’identification de ces kystes a été réalisée selon plusieurs critères notamment la taille, la forme, le nombre de noyaux, le contenu du cytoplasme, ceci à l'aide des planches de l’OMS (1994). Ainsi, le dénombrement a été effectué à l'aide de la formule proposée par Ajeagah et al.(2010). Le nombre (x) de kystes contenus dans 1L d'échantillon est obtenu par:

$$
\boldsymbol{x}=\boldsymbol{y} \frac{V x}{V y}
$$

Avec $V x=$ le volume du culot dans 1L d'échantillon, $V y=$ le volume du culot utilisé pour observer, $y$ le nombre de kystes observé dans $V y$. Les résultats sont donnés en nombre de kystes /L pour les échantillons d'eau.

\section{Analyse de donnée}

Test de corrélation de rang « $\mathbf{r}$ » de Spearman

La distribution étant anormale, les corrélations de rang de Spearman ont permis d'évaluer le degré de liaison entre les paramètres physicochimiques d'une part, et physico-chimiques et biologiques d'autre part. Les analyses ont été effectuées à l'aide du logiciel SPSS 16.0 et les résultats appréciés au seuil de sécurité de $99 \%(\mathrm{P}<0,01)$ et $95 \%(\mathrm{P}<0,05)$.

\section{Tests de comparaison des moyennes}

La variation spatiale des paramètres physico-chimiques et biologiques mesurés, a été testée à l’aide du test de Kruskal-Wallis associé au test de Mann-Whitney. Le test de Kruskal-Wallis a ainsi permis de déterminer si un paramètre varie de façon significative d'une station à une autre. Le test de Mann-Whitney appliqué aux paramètres qui varient d'une station à une autre a permis de déceler la source de variation. Les analyses ont été effectuées à l'aide du logiciel SPSS 16.0 et les résultats appréciés au seuil de sécurité de $95 \%(\mathrm{P}<0,05)$.

\section{Analyse en composantes principales (ACP)}

L'ACP a permis d'une part de mettre en évidence les relations de dépendance entre les différents paramètres mesurés et d’autre part, de déterminer les variables qui caractérisent chaque station. L'analyse a été réalisée à l’aide du logiciel XLSTAT.II.RESULTATS ET DISCUSSION

\section{Paramètres morphométriques et hydrologiques}

Le tableau III présente les caractéristiques morphométriques et hydrologiques des sources et puits, relevées au cours de cette étude. Les hauteurs des margelles fluctuent entre 0,10 m (NkP et EtP) et 0,70 m (MoP). Les diamètres varient de $0,7 \mathrm{~m}(\mathrm{NkP})$ à $1,28 \mathrm{~m}$ (MeP). Les épaisseurs 


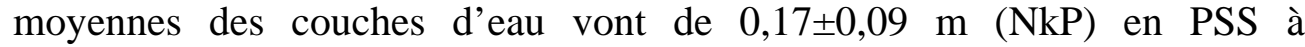
$1,87 \pm 0,06 \mathrm{~m}$ (MlS) en PSP. Les débits moyens d'écoulement d'eau varient de $0,00289 \pm 0,00027 \mathrm{~m}^{3} / \mathrm{s}$ en PSS (EtS) à $0,00425 \pm 0,00025 \mathrm{~m}^{3} / \mathrm{s}$ en PSP $(\mathrm{NkS})$. En effet, il ressort que $50 \%$ de puits étudiés ont les hauteurs de la margelle ne respectant pas les normes de l'OMS, qui recommande la construction d'une hauteur de margelle d'au moins 0,5 m.

Dans l'ensemble, le débit d'écoulement des sources et l'épaisseur de la colonne d'eau des puits ont été plus élevés en PSP. En effet, en saison de pluies, les eaux de pluies s'infiltrent et augment le niveau de la nappe. Dans ce même ordre d’idée, Gaston Lienou et al. (1984) ont montré qu'en saison de pluie, la nappe emmagasine d'importante quantité d'eau par le processus d'infiltration.

Tableau II: Caractéristiques morphométriques et hydrologiques des points d'échantillonnage

\begin{tabular}{|c|c|c|c|c|c|c|c|c|c|c|c|c|c|}
\hline \multicolumn{2}{|c|}{ Paramètres } & MoS & MoP & NkS & NkP & MeS & MeP & MIS & MIP & EtS & EtP & ObS & ObP \\
\hline \multicolumn{2}{|c|}{$\begin{array}{l}\text { Hauteur } \\
\text { margelle(m) }\end{array}$} & / & 0,7 & 1 & 0,1 & l & 0,5 & l & 0,65 & l & 0,1 & / & 0,15 \\
\hline \multicolumn{2}{|c|}{ Diamètre(m) } & 1 & 0,95 & 1 & 0,7 & 1 & 1,28 & 1 & 1,1 & 1 & 1,5 & 1 & 1,25 \\
\hline \multicolumn{2}{|c|}{ Hauteur (m) } & .1 & 1,9 & 1 & 0,7 & 1 & 2,25 & 1 & 2,15 & 1 & 2,3 & 1 & 2,1 \\
\hline EC(m) & \multirow{2}{*}{ PSP } & $\begin{array}{l}1,26 \pm \\
0,01\end{array}$ & $\begin{array}{l}0,47 \\
\pm \\
0,02\end{array}$ & 1 & $\begin{array}{l}0,34 \\
\pm \\
0,23\end{array}$ & / & $\begin{array}{l}1,58 \\
\pm \\
0,32 \\
\end{array}$ & $\begin{array}{l}1,87 \\
\pm \\
0,06\end{array}$ & $\begin{array}{l}1,23 \\
\pm \\
0,32 \\
\end{array}$ & l & $\begin{array}{l}1,14 \\
\pm \\
0,05\end{array}$ & l & $\begin{array}{l}1,48 \\
\pm \\
0,07\end{array}$ \\
\hline $\begin{array}{l}\text { D } \\
\left(\mathrm{m}^{3} / \mathrm{s}\right) 10^{-4}\end{array}$ & & 0 & I & \begin{tabular}{|l|}
42,5 \\
\pm \\
2,5 \\
\end{tabular} & / & $\begin{array}{l}38 \\
\pm \\
2,2\end{array}$ & l & 0 & / & $\begin{array}{l}29,1 \\
\pm \\
1,7\end{array}$ & l & $\begin{array}{l}30,2 \\
\pm \\
1,8\end{array}$ & / \\
\hline EC(m) & & $\begin{array}{l}1,18 \pm \\
0,01\end{array}$ & $\begin{array}{l}0,44 \\
\pm \\
0,05\end{array}$ & 1 & $\begin{array}{l}0,17 \\
\pm \\
0,09\end{array}$ & l & $\begin{array}{l}1,50 \\
\pm \\
0,42\end{array}$ & $\begin{array}{l}0,86 \\
\pm \\
0,22\end{array}$ & $\begin{array}{l}1,63 \\
\pm \\
0,66 \\
\end{array}$ & / & $\begin{array}{l}1,24 \\
\pm \\
0,05\end{array}$ & / & $\begin{array}{l}1,39 \\
\pm \\
0,05\end{array}$ \\
\hline $\begin{array}{l}\text { D } \\
\left(\mathrm{m}^{3} / \mathrm{s}\right) 10^{-4}\end{array}$ & PSS & I & / & $\begin{array}{l}39,4 \\
\pm \\
3,7\end{array}$ & l & $\begin{array}{l}30,6 \\
\pm \\
2,9\end{array}$ & / & l & / & $\begin{array}{l}28,9 \\
\pm \\
2,4\end{array}$ & / & $\begin{array}{l}30 \\
\pm \\
2,8 \\
\end{array}$ & I \\
\hline
\end{tabular}

EC: épaisseur de la couche d'eau; D: débit d'écoulement

\section{Paramètres physico-chimiques}

La valeur moyenne de la température $(24,88 \pm 0,81)^{\circ} \mathrm{C}$ se rapproche de celle de l'air $\left(25^{\circ} \mathrm{C}\right)$. Ceci pourrait s'expliquer par le fait que les sources et les puits étant ouverts, les rayons du soleil atteignent la nappe d'eau. A ce propos, Mbawala et al. (2010) révèlent que, les eaux souterraines ayant les températures proches de celles de l'air, indiquent l'ouverture du système aquifère. Ces résultats sont en accord avec ceux obtenus par Tuékam Kayo (2007) dans les eaux souterraines du bassin versant du Mfoundi et de la Mefou.

Les valeurs élevées des MES, de la turbidité et de la couleur ont été enregistrées respectivement dans les puits d’Etoug-ébé et Nkomkana (Tableau VII). Ces fortes valeurs pourraient être dues à leur mauvais état de protection. En effet, ces puits ne sont pas aménagés. Ils sont sans couvercles 
et n’ont pas de véritables margelles. Or, l’OMS (2011) recommande de construire des puits ayant une margelle à hauteur d'au moins 0,5 m munis d'une trappe mobile. En outre, les tests statistiques ont montré des corrélations significatives et positives entre les MES et d'autres paramètres physicochimiques tels que la couleur $(\mathrm{r}=0,886$; $\mathrm{p}=0,000)$ et la turbidité $(\mathrm{r}=$ 0,784; $\mathrm{p}=0,000)$.

Les valeurs de pH enregistrées au cours de l'étude, (Tableau VII) indiqueraient les eaux légèrement acides. Le $\mathrm{pH}$ acide au niveau des puits est semblable à l'étude faite par Nduka et al. (2007) à Warri au Nigeria ainsi que Debey et al. (2010) à Abomey-Calavi au Bénin, en dessous de la valeur normale qui est de 6,5 à 8,5 (OMS, 2012). Le caractère légèrement acide des eaux serait selon Nola et al. (2006) lié à la nature des sols traversés.

La valeur moyenne de la conductivité électrique est de 533,70 \pm 346,18 $\mu \mathrm{S} / \mathrm{cm}$. Ceci indiquerait les eaux moyennement minéralisées. De plus, les valeurs de la conductivité électrique sont très élevées dans les stations Melen 7 sources $(1178 \mu \mathrm{S} / \mathrm{cm})$ et Mokolo puits $(1139 \mu \mathrm{S} / \mathrm{cm})$, les caractérisant d’eaux très minéralisées selon Rodier et al. (2009). Cette forte conductivité enregistrée traduirait clairement que l'essentiel des substances ionisables dissoutes qui enrichissent ces milieux résulte de la minéralisation de la matière organique d'origine anthropique. Ainsi qu'à la nature équatoriale du sol de la ville de Yaoundé (Nola et al., 2006).

\section{Paramètres biologiques}

Au cours de cette étude, les observations des kystes de protozoaires flagellés ont permis de dénombrer une abondance totale de 14037 kystes appartenant à quatre (4) espèces. Il s’agit des kystes de Giardia intestinalis (28\%), Chilomastix mesnili (45\%), Enteromonas hominis (15\%) et Retortamonas intestinalis (12\%).

Les kystes de G. intestinalis (Figure 2A) ont une taille variant de 8 à $17 \mu \mathrm{m}$ de long. Ils sont de forme ovalaire ou ovoïde avec un contour lisse, assez mince et assez réfringent. Chaque kyste contient deux à quatre noyaux, des reliquats flagellés et un axostyle qui passe verticalement dans l'axe du kyste.

Le kyste de $C$. mesnili (Figure 2B) a une taille qui oscille entre 5 et $10 \mu \mathrm{m}$ de long. Il a la forme d'un citron ou d'une poire avec la coque externe lisse, épaisse, très nette et très réfringente. Le contenu est constitué d'un gros noyau excentré.

Le kyste d'E. hominis (Figure 2 C) a une forme ovalaire avec le contour très mince, peu net et peu réfringent. Sa taille fluctue entre 5 et 7 $\mu \mathrm{m}$ de long et son contenu est constitué de deux à quatre noyaux disposés aux extrémités du kyste. 
Le kyste de $R$. intestinalis (Figure $2 \mathrm{D}$ ) a une taille variant de 4 à 6 $\mu \mathrm{m}$ de long. En forme de poire, il est légèrement plus long que large. Son contenu présente un noyau allongé et entouré par un flagelle qui part de l'extrémité rétrécie du kyste et se dédouble en U.

L'analyse biologique montre la présence dans ces milieux, des kystes des protozoaires flagellés traduisant d'après l'OMS (2012) une contamination d'origine fécale.

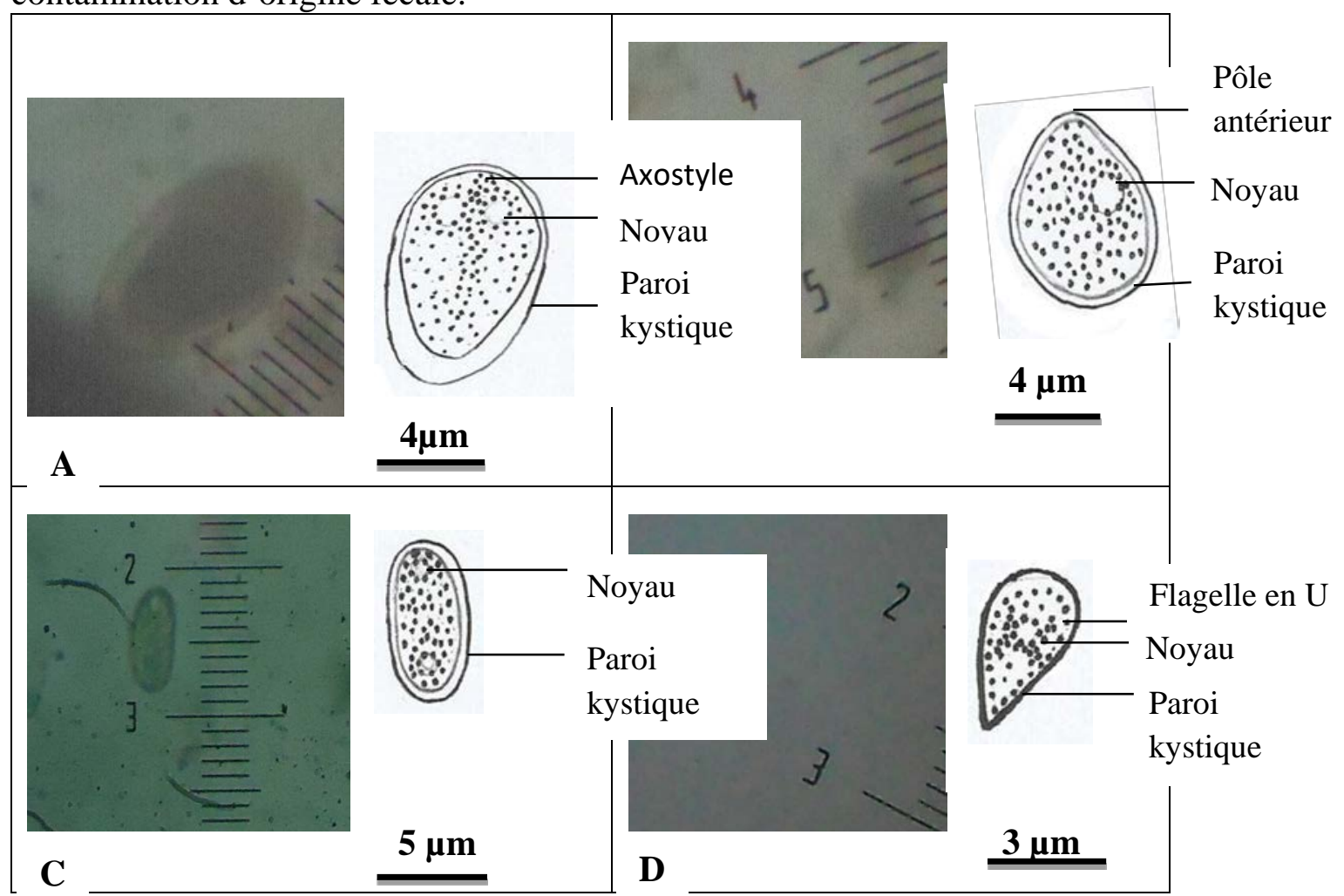

Figure 2: Photographies des kystes de différentes espèces de protozoaires flagellés observés : G. intestinalis (A), C. mesnili (B), E. hominis (C) et R. intestinalis (D).

\section{Impact des saisons et des paramètres physico-chimiques sur la dissémination des kystes \\ Tableau III: Corrélations entre quelques variables physico-chimiques et densités kystiques}

\begin{tabular}{|c|c|c|c|c|}
\hline \multirow{2}{*}{$\begin{array}{c}\text { Variables physico- } \\
\text { chimiques }\end{array}$} & \multicolumn{4}{|c|}{ Differentes especes de flagelles } \\
\cline { 2 - 5 } & $\begin{array}{c}\text { Giardia } \\
\text { intestinalis }\end{array}$ & $\begin{array}{c}\text { Chilomastöx } \\
\text { mesnili }\end{array}$ & $\begin{array}{c}\text { Enteromonas } \\
\text { hominis }\end{array}$ & $\begin{array}{c}\text { Retortamonas } \\
\text { intestinalis }\end{array}$ \\
\hline MES & $0,423^{*}$ & $0,426^{*}$ & 0,311 & $0,604^{* *}$ \\
\hline Couleur & $0,451^{*}$ & $0,485^{*}$ & $0,406^{*}$ & $0,619^{* *}$ \\
Turbidite & 0,28 & 0,339 & 0,251 & $0,435^{*}$ \\
\hline Oxydabilite & $0,467^{*}$ & $0,513^{*}$ & 0,287 & $0,518^{* *}$ \\
\hline O2 & 0,397 & $0,484^{*}$ & 0,283 & $0,494^{*}$ \\
\hline Nitrate & $-0,505^{*}$ & $-0,479^{*}$ & $-0,398$ & $-0,113$ \\
\hline
\end{tabular}

$*=$ corrélation significative à $\mathrm{p}<0,05 \quad * *=$ corrélation significative à $\mathrm{p}<0,01$ 
Sur le plan saisonnier, les densités les plus élevées des kystes ont été observées pendant la petite saison de pluies (Figure 3 \&Tableau VI) avec une densité moyenne de $212 \pm 216$ kystes/L. Ceci pourrait s’expliquer par le fait qu'en saison de pluies, la matière fécale drainée par les eaux de ruissellement, favorise les processus d'infiltration des kystes. Ces eaux, draineraient aussi divers déchets riches en matières organiques dans les sources et les puits qui sont dépourvus de margelles, et de couvercles. L’influence de la saison de pluie sur l'augmentation de la densité kystique est approuvée par l'OMS (2006) selon qui le taux de colonisation des eaux par les microorganismes pathogènes peut être favorisé par les précipitations. Les corrélations positives sont obtenues entre les MES, la turbidité et l'oxydabilité avec la densité des kystes. En effet, la contamination des eaux souterraines couplée à une forte teneur en MES favoriserait la dissémination des kystes. Les statistique ont montrés des corrélations significatives et positives entre les MES, la couleur, la turbidité et l'oxydabilité avec les kystes ( $\mathrm{p}<0,05)$. Selon Medema et al. (1998) ; Ajeagah et al. (2010), l'adhésion des kystes des parasites aux matières organiques en suspension favorise leur dissémination dans l'eau.

Les faibles densités observées pendant la petite saison sèche (Figure 3 \&Tableau VI) avec une densité moyenne de $81 \pm 94$ kystes/L, seraient imputables à la rareté des eaux de ruissellement pendant cette saison et à l'importance de l'activité minéralisatrice. En effet, les matières organiques stockées dans les eaux de puits et des sources sont progressivement transformées en éléments minéraux, qui pourraient constituer des agents stressants pour la survie des kystes. A ces propos, Ajeagah et al. (2007) soulignent que, de fortes concentrations en éléments minéraux peuvent augmenter l'inactivation des formes de résistance des organismes parasites. Ceci mettrait en exergue les corrélations négatives obtenues entre les nitrates et la densité des kystes $(\mathrm{p}<0,01)$.

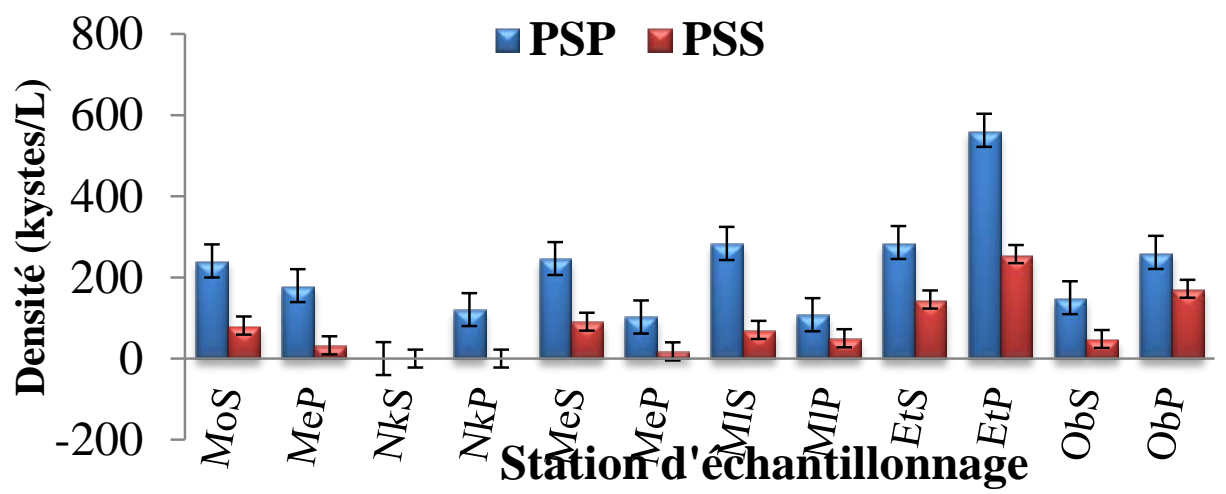

Figure 3: Variations spatiale et saisonnière des moyennes des densités des kystes des protozoaires flagellés pendant la période d'étude. 
La densité kystique élevée en PSP montre que, non seulement la contamination de ces puits et sources est exogène (par écoulement d'eau contaminée dans le puits, puisette ou corde souillée, action du vent), mais qu'elle serait due au processus d'infiltration des eaux de ruissellement et par les effluents souterrains des eaux des latrines pour celles qui ont une distance inferieure a 15 m (OMS) des sources et puits. Ceci s'expliquerait par le fait que les stations MoP, MeP et MIP en dépit de leurs margelles respectant la norme d’OMS (Tableau III), ont révélé les densités kystiques élevées en PSP. A ce propos, Hinden (2007) souligne que, l'eau constitue le moyen de transport des microorganismes et que le risque d'infiltration des polluants dépend donc de la quantité d'eau mise en jeu à l'endroit du dépôt. Dans le même sens, les travaux de Mbalawa et al. (2010) ont montré que, les kystes diffuseraient à travers l'eau des installations septiques vers les point d'eau d'une part, et que l'eau des puits serait contaminée à la suite d'infiltration des eaux de ruissellement d'autre part. La contamination de la nappe superficielle par les kystes de protozoaires pendant la période d'étude, corrobore ceux de Yousefi et al. (2009); Degbey et al. (2010) et Mbawala et al. (2010) qui ont noté une contamination de la nappe par les kystes de Giardia.

La forte abondance observée dans le puits Etoug-Ebé (3280 kystes) pendant les deux saisons serait due à une topographie favorable, la présence de faible margelle et d'une gestion hygiénique approximative de la puisette accentuée d'un défaut d'assainissement aux alentours de cette station. A ce propos, Hinden (2007) souligne que les conditions de stockage de la puisette et le type de construction permettent la contamination du puits.

\section{Risques sanitaires}

Les analyses ont montré une contamination des eaux de sources et de puits à hauteur de 91,67 \% des stations prospectées. Ces eaux utilisées très souvent pour les besoins ménagers, baignade, cuisine et boisson exposeraient les ménages à des risques sanitaires. A cet effet, les études faites au Cameroun (Nola et al., 1998; Pegnuité, 2004) ont montré que les populations s'exposent à des risques sanitaires à court terme en consommant les eaux de sources et de puits. Car la qualité de ces eaux se trouve le plus souvent affectée par une contamination d'origine fécale (Zamxaka et al., 2004). 
Tableau IV: Etat des kystes protozoaires flagellés entériques dans les sources et puits

\begin{tabular}{|c|c|c|ccc}
\hline \multicolumn{2}{|c|}{ STATIONS } & $\begin{array}{c}\text { Giardia } \\
\text { intertinafis }\end{array}$ & $\begin{array}{c}\text { Chilomastix } \\
\text { mesnifi }\end{array}$ & $\begin{array}{c}\text { Enteromonas } \\
\text { hominis }\end{array}$ & $\begin{array}{c}\text { Retortamonas } \\
\text { intestinalis }\end{array}$ \\
\hline \multirow{2}{*}{ Mokolo } & source & + & + & + & + \\
\cline { 2 - 6 } & puits & + & + & + & + \\
\hline \multirow{2}{*}{ Nkomkana } & source & - & - & + & + \\
& puits & + & + & + & + \\
\cline { 2 - 6 } Melen 1 & source & + & + & + & + \\
& puits & + & + & + & + \\
\hline \multirow{2}{*}{ Melen 7 } & source & + & + & + & + \\
& puits & + & + & + & + \\
\cline { 2 - 6 } Etoug-Ebé & source & + & + & + & + \\
\cline { 2 - 6 } & puits & + & + & + & + \\
& source & + & + & + & + \\
& puits & + & + & + \\
\hline
\end{tabular}

(-) absence de contamination par les kystes, $(+)$ contamination

\section{Caractérisation des stations d'échantillonnage}

L’Analyse en Composantes Principales réalisée sur les 14 paramètres physico-chimiques et 4 paramètres biologiques de 12 stations d'échantillonnage montre que, les deux premiers axes cumulent $58,47 \%$ de la variance totale (le premier axe explique $35,97 \%$ et le deuxième axe explique 22,49\%). Cette analyse a présenté un regroupement de l'ensemble des paramètres mesurés et des stations d'échantillonnage en 4 noyaux (Figure 4). Les noyaux I et II montrent un regroupement des paramètres physico-chimiques tels que la conductivité électrique, les TDS, l'alcalinité, les nitrates, les orthophosphates et la température. Ces paramètres caractérisent la station NkP, MeP, MlP et ObS. La station NkS s'écarte des autres par une absence totale des kystes pendant toute la période d'échantillonnage. En effet, la source Nkomkana est de type rhéocrène avec un débit d'écoulement élevé et bénéficie aussi d'un meilleur système de protection construit en béton et menu d'un robinet.

En outre, l'ACP a montré que l'axe F2 est fortement corrélé du côté positif avec la conductivité électrique, les TDS, l'alcalinité, les nitrates et les orthophosphates autour du noyau II. Cet axe exprime la minéralisation. Dans les noyaux III et IV, les paramètres physico-chimiques tels que les MES, la couleur, la turbidité, l'oxydabilité, l'oxygène, le chlore libre et le $\mathrm{pH}$ ont de fortes liaisons avec les kystes et caractérisent les stations MoS, MoP, MeS, MlS, EtS et ObP. La station puits Etoug-Ebé se distingue des autres par une forte dynamique d'abondance des kystes (noyau IV). En effet, l'ACP a montré que l'axe F1 est fortement corrélé (35,97\%) du côté positif avec l'oxydabilité, les MES, le couleur, la turbidité et les matières organiques d'une part et aux différents densités kystiques analysés d'autre part autour du noyau III. Cet axe exprime à la fois les pollutions 
organiques et biologiques caractérisant les stations MoS, MoP, MeS, MlS, EtS et ObP.

Biplot (axes F1 et F2 : 58,47\%)

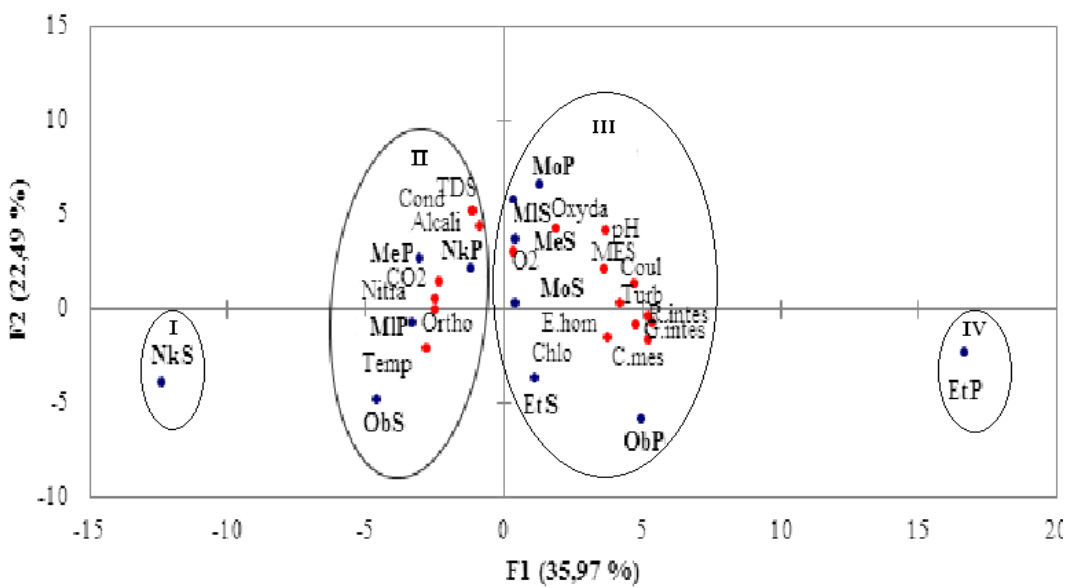

Figure 4: ACP des stations d'échantillonnage en fonction des variables mesurées.

Temp : Température ; pH : Potentiel Hydrogène ; Cond : Conductivité Electrique ; $\mathrm{CO}_{2}$ : Gaz Carbonique ; $\mathrm{O}_{2}$ : Oxygène Dissous ; Alca : Alcalinité ; TDS : Solides Totaux Dissous ; Turb : Turbidité ; Coul : Couleur ; MES : Matières En Suspension ; $\mathrm{PO}_{4}{ }^{3-}$ : Orthophosphates ; $\mathrm{NO}_{3}{ }^{-}$: Nitrate ; Oxyda : Oxydabilité ; G. intes : intestinalis ; 1 E. hom : Enteromonas hominis ; Giardia intestinalis ; C.mes : Chilomastix mesnili ; R. intes : Retortamonas.

La forte teneur du chlore libre dans les puits d Etoug-Ebé et Obili (Tableau V) montre l'intention des ménages à traiter ces eaux avant toute utilisation. Or la présence d'abondances relatives élevées respectivement 3280 kystes et 1735 kystes malgré les fortes concentrations du chlore libre dans ces stations montrerait une résistance des kystes à ce désinfectant. Ces résultats corroborent ceux de Markell et al. (1999) qui révèlent que, les kystes de Giardia en particulier ne sont pas détruits à des doses répétées d'hypochlorite de sodium dans l'eau de boisson.

Tableau V: Effets d'hypochlorite de sodium sur les kystes

\begin{tabular}{lllllllllllll}
\hline Paramètres & \multicolumn{2}{l}{ Sources } & & & \multicolumn{1}{c}{ Puits } & & & & \\
& MoS & NkS & MeS & MIS & EtS & ObS & MoP & NkP & MeP & MIP & EtP & ObP \\
\hline $\begin{array}{l}\text { Chlore } \\
\text { libre }\end{array}$ & 9,20 & 0,29 & 11,95 & 0,75 & 1,00 & 1,92 & 0,88 & 0,89 & 12,73 & 7,52 & 19,06 & 19,56 \\
\hline $\begin{array}{l}\text { Abondance } \\
\text { relative }\end{array}$ & 1289 & 0 & 1356 & 117 & 1727 & 793 & 850 & 483 & 841 & 632 & 3280 & 1956 \\
\hline
\end{tabular}


Tableau VI: Densités des kystes de différentes espèces de flagellés identifiés dans les sources et puits pendant la période d'étude (en kystes/L)

\begin{tabular}{|c|c|c|c|c|c|c|}
\hline Stations & Paramètres & G.intestinalis & C.mesnili & E.hominis & Rintestinalis & Densité totale \\
\hline \multirow[b]{3}{*}{ Mos } & PSS & 84 & 200 & 42 & 0 & 326 \\
\hline & Moyenne & 172 & 289 & 105 & 79 & \\
\hline & Ecart-type & 124 & 125 & 89 & 112 & \\
\hline \multirow[b]{4}{*}{ MoP } & PSP & 236 & 285 & 140 & 58 & 719 \\
\hline & PSS & 91 & 28 & 12 & 0 & 131 \\
\hline & Moyenne & 164 & 157 & 76 & 29 & \\
\hline & Ecart-type & 102 & 182 & 91 & 41 & \\
\hline \multirow[b]{4}{*}{$\mathrm{NkS}$} & PSP & 0 & 0 & 0 & 0 & 0 \\
\hline & PSS & 0 & 0 & 0 & 0 & 0 \\
\hline & Moyenne & 0 & 0 & 0 & 0 & \\
\hline & Ecart-type & 0 & 0 & 0 & 0 & \\
\hline \multirow[b]{4}{*}{$\mathrm{NkP}$} & PSP & 139 & 198 & 29 & 117 & 483 \\
\hline & PSS & 0 & 0 & 0 & 0 & 0 \\
\hline & Moyenne & 70 & 99 & 15 & 59 & \\
\hline & Ecart-type & 98 & 140 & 20 & 83 & \\
\hline \multirow[b]{4}{*}{ Mes } & PSP & 275 & 453 & 156 & 102 & 986 \\
\hline & PSS & 106 & 119 & 139 & 0 & 364 \\
\hline & Moyenne & 191 & 286 & 148 & 51 & \\
\hline & Ecart-type & 120 & 237 & 12 & 72 & \\
\hline \multirow[b]{4}{*}{ MeP } & PSP & 151 & 138 & 0 & 121 & 410 \\
\hline & YSS & 28 & 23 & 0 & 20 & 71 \\
\hline & Moyenne & 90 & 81 & 0 & 71 & \\
\hline & Ecart-type & 86 & 82 & 0 & 71 & \\
\hline \multirow[b]{4}{*}{ MIS } & PSP & 300 & 334 & 222 & 279 & 1135 \\
\hline & PSS & 104 & 178 & 0 & 0 & 282 \\
\hline & Moyenne & 202 & 256 & 111 & 140 & \\
\hline & Ecart-type & 139 & 110 & 157 & 197 & \\
\hline \multirow[b]{4}{*}{ MIP } & PSP & 70 & 201 & 62 & 99 & 432 \\
\hline & PSS & 102 & 53 & 45 & 0 & 200 \\
\hline & Moyenne & 86 & 127 & 54 & 50 & \\
\hline & Ecart-type & 23 & 105 & 12 & 70 & \\
\hline
\end{tabular}

\section{Conclusion}

Ette étude a permis de caractériser les formes de résistance de protozoaires flagellés intestinaux dans les eaux des sources et des puits de quelques quartiers (Mokolo, Nkomkana, Melen I, Melen 7, Etoug-ébé et Obili) de la ville de Yaoundé. L’analyse des résultats révèle que ces eaux sont légèrement acides, moyennement minéralisées et riches en matières en suspension. En outre, la densité kystique a été plus élevée en PSP qu'en PSS, mettant en exergue le rôle prépondérant de l'eau dans le transport des kystes. Les analyses statistiques ont révélé des corrélations positives et significatives entre la densité des kystes et certains paramètres physico-chimiques comme les MES, la turbidité, la couleur et l'oxydabilité, d'une part, et négativement corrélé aux nitrates d'autre part, au seuil de 5\%. La station Etoug-ébé puits 
s’est caractérisée par de fortes charges parasitaires contrairement à la station Nkomkana source qui a un meilleur système de protection et dépourvu des kystes. La présence de ces organismes dans ces milieux indique une mauvaise qualité de l'eau de consommation à des risques sanitaires. Afin de limiter les transmissions des maladies liées aux protozoaires flagellés, il serait important de construire les puits qui respectent les normes de l'OMS, de traiter ces eaux par les méthodes simples et adéquates (chloration, ébullition et SODIS), aussi de participer à l’assainissement de leur milieu.

\section{References:}

1. Ajeagah G. A., Njiné T., Nola M., Foto M. S. \& Wouafo N.M. 2007. Evaluation de l'abondance des formes de résistance de deux protozoaires pathogènes (Giardia sp. et Cryptosporidium sp.) dans deux biotopes aquatiques de Yaoundé (Cameroun). Cahier d'Etudes et de Recherche Francophones/Santé, 17(3): 167-172.

2. Ajeagah G. A., Njine T., Bilong Bilong C. F., Foto S.M., Wouafo N. M., Nola M., Di G. G. D. \& Huw S. 2010. Seasonal Distribution of Enteric Opportunistic Cryptosporidium Spp. Oocysts and Giardia Spp. Cysts in a tropical water basin. Cameroon. Water, 2: 44-57.

3. Apha. 1998. Standard method for examination of water and wastewater. American Public Health Association, 20th edn, Washington, $1150 \mathrm{p}$.

4. Aubry P., Chapoy P. \& Gras C. 1986. Syndrome de malabsorption et giardiase. Médecine d'Afrique Noire, 33: 95-100.

5. Belghiti M.L., Chahlaoui A., Bengoumi D. \& EL Moustaine R. 2013. Étude de la qualité physicochimique et bactériologique des eaux souterraines de la nappe plio-quaternaire dans la région de Meknès. Larhyss Journal, 14: 21-36.

6. Bertrand I., Gantzer C., Chesnot T. \& Schwartzbrod J. 2004. Improved specificity for Giardia lamblia cystsin quantification \& wastewater by development of areal-tim PCR. Journal Microbiology and Methods, 57: 41-53.

7. Camwater. 2011. Insuffisance de la capacité de production et de distribution. Mutation, $8 \mathrm{p}$

8. Degbey C. Makoutodem., Ouendo E.M. \& Brouwer C. 2010. Pollution physicochimique et microbiologique de l'eau des puits dans la Commune d'Abomey-Calavi au Bénin en 2009. International Journal of Biological and Chemical Sciences, 4(6): 2257-2271.

9. Dejoux C. 1988. La pollution des eaux continentales africaines : Expérience acquise, situation actuelle et perspectives. ORSTOM, Paris, 513 p. 
10. Euzeby J. 2002. L’épidémiologie de la giardiose humaine. Scientia Parasitologica, 1: 14-128.

11. Faust E.C., D’Antni J.S., Odom V., Miller M.J., Peres C., Sawitz W., Thomen L.F., Tobie J. \& Walker J.H. 1938. A critical study of clinical laboratory technics for the diagnosis of protozoan cysts and helminth eggs in feces. American joumal of tropical medicine and hygiene, 18: 169-18.

12. Foster S. S. D. 2001. The interdependence of groundwater and urbanization in rapidly developing cities. Urban water, 3: 185-192.

13. Gaston L., Gil M., Jean- Emmanuel P., Eric S., Helene L.N., Daniel S., Georges E.E. \& Alain D. 2002. Changement des régimes hydrologiques dans les rivières du Sud-Cameroun: un impact de la variabilité alimentaire en zone équatoriale. HyroScience Montpellier, 158-168.

14. Hinden C. 2007. La contamination de l'eau de boisson dans le village de Bengou (Niger). Mémoire de Licence, Institut de géographie Faculté des géosciences et de l’environnement de Lausanne, $62 \mathrm{p}$.

15. INS (Institut National de la Statistique). 2013. Étude pilote sur la pollution des eaux de surface et souterraines à Yaoundé et son impact sur la santé des populations riveraines. Rapport technique publié à Yaoundé, Hanovre, Bonn, 335 p.

16. Johan A., Jon J-H., Emma R., Karin T. \& Staffan G. 2010. Behind the smile: cell biology and disease mechanism of giardia species. Nature Reviews Microbiology, 8: 413-422

17. Lacoste R. 2009. Les parasites intestinaux chez le macaque crabier (Macaca fascicularis): Etude expérimentale et recommandations pour la diagnose et la gestion des rhizoflagelles et des ciliés. Thèse de Doctorat vétérinaire, Faculté de Médecine de Créteil/Ecole Nationale Vétérinaire d'Alfor, 237 p.

18. Markell E.K., John D.T. \& krotoski W. 1999. A medical parasitology, 8thed W.B. Saunders Company. Philadelphia, 22-42.

19. Mbawala A., Abdou., Ngassoum M.B. \& Ouendo. 2010. Pollution physico-chimique et microbiologique de l'eau de puits de DengNgaoundéré au Cameroun. International Journal of Biological and Chemical Sciences, 4(6): 1962-1975.

20. Medema G. J., Schets F. M., Teunis P. F. M. \& Havelaar N. H. 1998. Sedimentation of free and attached Cryptosporidium oocysts and Giardia in water. Appleid Environnemental Microbiology, 64: 4460-4466. 
21. Nduka J.K. \& Orisakwe O.E. 2007. Heavy metal levels and physicochemical quality of potable water supply in Warri, Nigeria. Announcement Chemistry, 97(9): 867-74.

22. Nkhuwa D. C. 2003. Human activities and threats of chronic epidemics in a fragile geologic environment. Physics and Chemistry of the Earth, 28: 1139-1149.

23. Nola M., Njine T., Monkiedje A., Sikati FV. \& Djuikom E. 1998. Qualité bactériologique des eaux des sources et des puits de Yaoundé (Cameroun). Cahiers. Santé, 8(5): 330-336.

24. Nola M., Njine T., Kemka N., Zébazé Togouet S. H., Foto Menbohan S., Monkiedje A., Servais P., Messouli M. \& Boutin Cl. 2006. Retention of staphylococci and total coliforms during wastewater percolation through equatorial soil in Central Africa: The role of the soil column near soil surface and that closely above groundwater table. Water, Air and Soil Pollution, 171: 253-271.

25. OMS. 1994. Planches pour le diagnostique des parasites intestinaux .Genève, 29 p.

26. OMS. 2003. L'Eau et la santé : Directives de l'OMS sur la qualité de l'eau de boisson 13 p.

27. OMS. 2006. Directives de la qualité pour l'eau de boisson, critères d'hygiène et documentation à l'appui Volume 2.Organisation Mondiale de la Santé. $2^{\text {ème }}$ édition, 1050 p.

28. OMS. 2011. Guidelines for Drinking-water Quality. Fourth edition, WHO Library Cataloguing-in-Publication Data, 541 p.

29. OMS. 2012. Directives OMS pour l'utilisation sans risque des eaux usées, des excréta et des eaux ménagères. Utilisation des eaux usées et des excréta en aquaculture, Genève, 63 p.

30. Pegnuite N. 2004. Evaluation de la qualité microbiologique des eaux de puits de Bépanda. Mémoire de maîtrise de Biologie Animale. Université de Douala, 32 p.

31. Pelletier J. L. 1969. Données générales sur la répartition des principaux types de sol de la région de Yaoundé. Document Orstom, 25 p.

32. Petithory J.C., Ardoin-Guidon F. \& Chaumeil C. 1998. Cahier de formation - Biologie médicale; Amibes et flagellés intestinaux : amibes oculaires, leur diagnostic microscopique, 11, 237 p.

33. Raccurt C.P., Pannier S.C., Eyma E., Verdier R.I., Totet A. \& Pape J.W. 2006. Enteric parasites and AIDS in Haiti. Utility of detection and treatment of parasites in family members. Médecine Tropicale, (4), 461 p.

34. Rodier J., Legube B. \& Merlet N. 2009. L'analyse de l'eau. $9^{\mathrm{e}}$ édition, Dunod, Paris, 1526 p. 
35. Suchel B. 1972. La répartition des pluies et des régimes pluviométriques au Cameroun. Travaux et documents de géographie tropicale, 5: 1-288.

36. Tuekam Kayo R. P. 2007. Stygofaune de deux bassins versants souterrains contigus de la région de Yaoundé: influence de la pression Anthropique. Mémoire de DEA. Faculté des Sciences. Université de Yaoundé I, Cameroun, 69 p.

37. Wolfe M. S. 1992. Giardiasis. Clinical Microbiology Review, 5(1): 93-100.

38. Yousefi Z., Ziaei hezarjaribi H., Enayati A. A. \& Mohammadpoor R. A. 2009. Parasitic contamination of wells drinking water in Mazandaran province Iran. Journal of Environmental Health In Science and Engineering, 6(4): 241-246.

39. Zamxaka M., Pironcheva G., Muyima N., Pironcheva G. \& Muyima N. 2004. Microbiological and physicochemical assessment of the quality of domestic water sources in selected rural communities of the Eastern Cape Province, South Africa. Water, SA, 30: 333-340. 


\begin{tabular}{|c|c|c|c|c|c|c|c|c|c|c|c|c|c|c|c|}
\hline $\begin{array}{l}\text { Stati } \\
\text { ons }\end{array}$ & $\begin{array}{l}\text { Sais } \\
\text { ons }\end{array}$ & $\begin{array}{l}\text { Tempéra } \\
\text { ture }\end{array}$ & MES & Couleur & $\begin{array}{l}\text { Turbidit } \\
\text { é }\end{array}$ & $\mathrm{pH}$ & $\begin{array}{l}\text { Conductivi } \\
\text { té } \\
\text { électrique }\end{array}$ & TDS & $\begin{array}{l}\text { Oxydab } \\
\text { ilité }\end{array}$ & $\mathrm{O}_{2}$ & $\mathrm{CO}_{2}$ & Alcalinité & $\mathrm{NO}_{3}{ }^{-}$ & $\mathrm{PO}_{4}{ }^{3-}$ & $\begin{array}{l}\text { Chlore } \\
\text { libre }\end{array}$ \\
\hline \multirow[b]{2}{*}{ MoS } & PSP & $\begin{array}{l}24,38 \\
\pm 0,95\end{array}$ & $\begin{array}{l}12,00 \pm 2, \\
83\end{array}$ & $\begin{array}{l}20,00 \pm 8 \\
49\end{array}$ & $\begin{array}{l}5,40 \\
\pm 2,26\end{array}$ & $\begin{array}{l}6,03 \pm \\
0,48\end{array}$ & $\begin{array}{l}642,50 \pm 24 \\
1,31\end{array}$ & $\begin{array}{l}321,00 \pm 1 \\
19,85\end{array}$ & $\begin{array}{l}4,45 \pm 1 \\
14\end{array}$ & $\begin{array}{l}7,50 \pm \\
2,92\end{array}$ & $\begin{array}{l}24,20 \pm \\
9,35\end{array}$ & $\begin{array}{l}89,00 \pm 82, \\
08\end{array}$ & $\begin{array}{l}5,85 \pm 6 \\
15\end{array}$ & $\begin{array}{l}2,18 \pm \\
2,91\end{array}$ & $\begin{array}{l}13,10 \pm \\
5,35\end{array}$ \\
\hline & PSS & $\begin{array}{l}24,25 \\
\pm 0,35\end{array}$ & $\begin{array}{l}7,50 \\
\pm 5,07\end{array}$ & $\begin{array}{l}15,75 \pm 10 \\
, 14\end{array}$ & $\begin{array}{l}4,58 \pm 3,1 \\
1\end{array}$ & $\begin{array}{l}6,17 \pm \\
0,10\end{array}$ & $\begin{array}{l}532,00 \pm 48, \\
08\end{array}$ & $\begin{array}{l}265,00 \pm 2 \\
5,46\end{array}$ & $\begin{array}{l}3,36 \pm 1 \\
12\end{array}$ & $\begin{array}{l}4,30 \pm \\
0,57\end{array}$ & $\begin{array}{l}17,60 \pm \\
2,49\end{array}$ & $\begin{array}{l}24,00 \pm 5,6 \\
6\end{array}$ & $\begin{array}{l}4,50 \pm 0 \\
00\end{array}$ & $\begin{array}{l}0,24 \pm \\
0,00\end{array}$ & $\begin{array}{l}5,30 \pm 1, \\
84\end{array}$ \\
\hline \multirow[b]{2}{*}{ MoP } & PSP & $\begin{array}{l}25,38 \\
\pm 2,93 \\
\end{array}$ & $\begin{array}{l}26,50 \pm 6, \\
36\end{array}$ & $\begin{array}{l}49,50 \\
\pm 3,54 \\
\end{array}$ & $\begin{array}{l}10,42 \\
\pm 2,38 \\
\end{array}$ & $\begin{array}{l}6,56 \pm \\
0,35 \\
\end{array}$ & $\begin{array}{l}841,25 \pm 22 \\
2,72\end{array}$ & $\begin{array}{l}420,00 \pm 1 \\
11,75\end{array}$ & $\begin{array}{l}5,98 \pm 5, \\
23\end{array}$ & $\begin{array}{l}8,55 \pm \\
3,67 \\
\end{array}$ & $\begin{array}{l}24,20 \pm \\
6,49 \\
\end{array}$ & $\begin{array}{l}68,00 \pm 34 \\
91\end{array}$ & $\begin{array}{l}3,45 \pm 0, \\
07\end{array}$ & $\begin{array}{l}0,36 \pm \\
0,20 \\
\end{array}$ & $\begin{array}{l}1,35 \pm 0, \\
24\end{array}$ \\
\hline & PSS & $\begin{array}{l}24,75 \\
\pm 0,35\end{array}$ & $\begin{array}{l}24,75 \pm 1 \\
6,50\end{array}$ & $\begin{array}{l}47,00 \pm 25 \\
, 63\end{array}$ & $\begin{array}{l}8,51 \pm 7,6 \\
9\end{array}$ & $\begin{array}{l}6,62 \pm \\
0,10\end{array}$ & $\begin{array}{l}1 \\
242,50 \pm 26 \\
2,34 \\
\end{array}$ & $\begin{array}{l}521,50 \pm 9 \\
19\end{array}$ & $\begin{array}{l}3,46 \pm 1 \\
53\end{array}$ & $\begin{array}{l}4,10 \pm \\
0,28\end{array}$ & $\begin{array}{l}22,00 \pm \\
3,73\end{array}$ & $\begin{array}{l}174,00 \pm 3 \\
3,94\end{array}$ & $\begin{array}{l}3,70 \pm 0 \\
00\end{array}$ & $\begin{array}{l}0,41 \pm \\
0,00\end{array}$ & $\begin{array}{l}0,42 \pm 0, \\
40\end{array}$ \\
\hline \multirow[b]{2}{*}{$\mathrm{NkS}$} & PSP & $\begin{array}{l}26,23 \\
\pm 0,63\end{array}$ & $\begin{array}{l}0,00 \pm 0,0 \\
0\end{array}$ & $\begin{array}{l}0,00 \pm 0,0 \\
0\end{array}$ & $\begin{array}{l}0,00 \pm 0,0 \\
0\end{array}$ & $\begin{array}{l}5,17 \pm \\
0,67\end{array}$ & $\begin{array}{l}418,25 \pm 65 \\
58\end{array}$ & $\begin{array}{l}208,75 \pm 3 \\
0,99\end{array}$ & $\begin{array}{l}0,33 \pm 0, \\
10\end{array}$ & $\begin{array}{l}5,30 \pm \\
1,37\end{array}$ & $\begin{array}{l}27,72 \pm \\
2,21\end{array}$ & $\begin{array}{l}114,00 \pm 7 \\
7,34\end{array}$ & $\begin{array}{l}5,50 \pm 1 \\
70\end{array}$ & $\begin{array}{l}2,13 \pm \\
2,28\end{array}$ & $\begin{array}{l}0,22 \pm 0, \\
04\end{array}$ \\
\hline & PSS & $\begin{array}{l}25,25 \\
\pm 0,35\end{array}$ & $\begin{array}{l}0,00 \pm 0,0 \\
0\end{array}$ & $\begin{array}{l}0,00 \\
\pm 0,00\end{array}$ & $\begin{array}{l}0,00 \pm 0,0 \\
0\end{array}$ & $\begin{array}{l}5,13 \pm \\
0,06\end{array}$ & $\begin{array}{l}484,50 \pm 13, \\
44\end{array}$ & $\begin{array}{l}240,50 \pm 3 \\
54\end{array}$ & $\begin{array}{l}0,45 \pm 0 \\
49\end{array}$ & $\begin{array}{l}3,90 \pm \\
0,42\end{array}$ & $\begin{array}{l}23,12 \pm \\
4,64\end{array}$ & $\begin{array}{l}19,00 \pm 12 \\
73\end{array}$ & $\begin{array}{l}9,80 \pm 0 \\
00\end{array}$ & $\begin{array}{l}1,94 \pm \\
0,00\end{array}$ & $\begin{array}{l}0,35 \pm 0, \\
07\end{array}$ \\
\hline \multirow[b]{2}{*}{$\mathrm{NkP}$} & PSP & $\begin{array}{l}25,13 \\
\pm 0,25\end{array}$ & $\begin{array}{l}44,75 \pm 4 \\
1,56\end{array}$ & $\begin{array}{l}47,75 \\
\pm 21,08\end{array}$ & $\begin{array}{l}17,61 \\
\pm 23,37\end{array}$ & $\begin{array}{l}6,09 \pm \\
0,67\end{array}$ & $\begin{array}{l}\text { 406,75 } \pm 85 \\
92\end{array}$ & $\begin{array}{l}189,75 \pm 4 \\
4,21\end{array}$ & $\begin{array}{l}8,15 \pm 2, \\
92\end{array}$ & $\begin{array}{l}6,65 \pm \\
3,96\end{array}$ & $\begin{array}{l}13,87 \pm \\
3,39\end{array}$ & $\begin{array}{l}203,00 \pm 2 \\
86,73\end{array}$ & $\begin{array}{l}8,30 \pm 8, \\
34\end{array}$ & $\begin{array}{l}2,19 \pm \\
1,45\end{array}$ & $\begin{array}{l}0,65 \pm 1, \\
07\end{array}$ \\
\hline & PSS & $\begin{array}{l}24,30 \pm 0, \\
42\end{array}$ & $\begin{array}{l}8,50 \\
\pm 2,12 \\
\end{array}$ & $\begin{array}{l}10,50 \\
\pm 3,54 \\
\end{array}$ & $\begin{array}{l}10,09 \\
\pm 11,89 \\
\end{array}$ & $\begin{array}{l}6,65 \pm \\
0,42 \\
\end{array}$ & $\begin{array}{l}302,00 \pm 2,8 \\
3 \\
\end{array}$ & $\begin{array}{l}151,50 \pm 0, \\
71\end{array}$ & $\begin{array}{l}4,84 \pm 0, \\
42\end{array}$ & $\begin{array}{l}4,35 \pm \\
0,07\end{array}$ & $\begin{array}{l}22,88 \pm \\
2,49\end{array}$ & $\begin{array}{l}21,00 \pm 1,4 \\
1\end{array}$ & $\begin{array}{l}17,20 \pm \\
0,00\end{array}$ & $\begin{array}{l}1,54 \pm \\
0,00\end{array}$ & $\begin{array}{l}1,14 \pm 0, \\
47 \\
\end{array}$ \\
\hline \multirow[b]{2}{*}{$\mathrm{MeS}$} & PSP & $\begin{array}{l}23,88 \pm 1, \\
03\end{array}$ & $\begin{array}{l}16,25 \pm 1 \\
6,40\end{array}$ & $\begin{array}{l}24,25 \\
\pm 20,25 \\
\end{array}$ & $\begin{array}{l}4,04 \pm 1,5 \\
6\end{array}$ & $\begin{array}{l}6,32 \pm \\
0,47 \\
\end{array}$ & $\begin{array}{l}844,50 \pm 49, \\
82\end{array}$ & $\begin{array}{l}422,25 \pm 2 \\
5,04\end{array}$ & $\begin{array}{l}8,63 \pm 8, \\
43\end{array}$ & $\begin{array}{l}7,93 \pm \\
3,52 \\
\end{array}$ & $\begin{array}{l}28,60 \pm \\
2,64\end{array}$ & $\begin{array}{l}87,50 \pm 36, \\
38\end{array}$ & $\begin{array}{l}4,80 \pm 0 \\
00\end{array}$ & $\begin{array}{l}1,78 \pm \\
2,28 \\
\end{array}$ & $\begin{array}{l}11,45 \pm \\
0,94\end{array}$ \\
\hline & PSS & $\begin{array}{l}24,25 \\
\pm 0,06\end{array}$ & $\begin{array}{l}8,50 \pm 2,1 \\
2\end{array}$ & $\begin{array}{l}7,50 \pm 6,3 \\
6\end{array}$ & $\begin{array}{l}3,00 \\
\pm 2,83\end{array}$ & $\begin{array}{l}6,52 \pm \\
0,11\end{array}$ & $\begin{array}{l}818,00 \pm 1,4 \\
1\end{array}$ & $\begin{array}{l}409,00 \pm 0, \\
00\end{array}$ & $\begin{array}{l}3,36 \pm 2 \\
79\end{array}$ & $\begin{array}{l}4,35 \pm \\
0,35\end{array}$ & $\begin{array}{l}22,00 \pm \\
3,73\end{array}$ & $\begin{array}{l}40,00 \pm 0,0 \\
0\end{array}$ & $\begin{array}{l}4,30 \pm 0 \\
00\end{array}$ & $\begin{array}{l}2,26 \pm \\
0,00\end{array}$ & $\begin{array}{l}12,45 \pm \\
1,77\end{array}$ \\
\hline \multirow[b]{2}{*}{$\mathrm{MeP}$} & PSP & $\begin{array}{l}25,38 \\
\pm 0,95\end{array}$ & $\begin{array}{l}18,00 \pm 1 \\
9,70\end{array}$ & $\begin{array}{l}19,00 \\
\pm 12,25\end{array}$ & $\begin{array}{l}6,71 \pm 6,0 \\
4\end{array}$ & $\begin{array}{l}5,96 \pm \\
0,18\end{array}$ & $\begin{array}{l}823,50 \pm 10 \\
4,55\end{array}$ & $\begin{array}{l}409,00 \pm 4 \\
8,46\end{array}$ & $\begin{array}{l}7,55 \pm 4, \\
47\end{array}$ & $\begin{array}{l}8,58 \pm \\
3,62\end{array}$ & $\begin{array}{l}29,44 \pm \\
3,30\end{array}$ & $\begin{array}{l}65,50 \pm 65 \\
43\end{array}$ & $\begin{array}{l}5,00 \pm 1, \\
98\end{array}$ & $\begin{array}{l}1,17 \pm \\
1,23\end{array}$ & $\begin{array}{l}14,55 \pm \\
4,07\end{array}$ \\
\hline & PSS & $\begin{array}{l}24,50 \\
\pm 0,71 \\
\end{array}$ & $\begin{array}{l}17,00 \pm 2, \\
83\end{array}$ & $\begin{array}{l}18,50 \\
\pm 9,19 \\
\end{array}$ & $\begin{array}{l}6,50 \pm 2,1 \\
2\end{array}$ & $\begin{array}{l}6,03 \pm \\
0,11\end{array}$ & $\begin{array}{l}780,00 \pm 0,0 \\
0\end{array}$ & $\begin{array}{l}390,00 \pm 0 \\
00\end{array}$ & $\begin{array}{l}2,67 \pm 0, \\
98\end{array}$ & $\begin{array}{l}3,70 \pm \\
0,71 \\
\end{array}$ & $\begin{array}{l}23,76 \pm \\
3,73\end{array}$ & $\begin{array}{l}30,00 \pm 11 \\
31\end{array}$ & $\begin{array}{l}12,10 \pm \\
0,00\end{array}$ & $\begin{array}{l}0,70 \pm \\
0,00\end{array}$ & $\begin{array}{l}10,90 \pm \\
0,14\end{array}$ \\
\hline \multirow[b]{2}{*}{ MlS } & PSP & $\begin{array}{l}25,88 \\
\pm 0,63 \\
\end{array}$ & $\begin{array}{l}10,25 \pm 9, \\
88\end{array}$ & $\begin{array}{l}19,75 \\
\pm 4,65 \\
\end{array}$ & $\begin{array}{l}2,50 \\
\pm 3,03\end{array}$ & $\begin{array}{l}6,58 \pm \\
0,51\end{array}$ & $\begin{array}{l}985,75 \pm 32 \\
4,43\end{array}$ & $\begin{array}{l}492,50 \pm 1 \\
62,51\end{array}$ & $\begin{array}{l}10,76 \pm \\
2,77\end{array}$ & $\begin{array}{l}5,00 \pm \\
0,99\end{array}$ & $\begin{array}{l}14,29 \pm \\
3,88\end{array}$ & $\begin{array}{l}121,50 \pm 9 \\
3,40\end{array}$ & $\begin{array}{l}4,15 \pm 4, \\
17\end{array}$ & $\begin{array}{l}1,20 \pm \\
1,29\end{array}$ & $\begin{array}{l}0,19 \pm 0, \\
02\end{array}$ \\
\hline & PSS & $\begin{array}{l}24,00 \\
\pm 0,00 \\
\end{array}$ & $\begin{array}{l}2,50 \\
\pm 0,71 \\
\end{array}$ & $\begin{array}{l}16,00 \\
\pm 16,97 \\
\end{array}$ & $\begin{array}{l}1,06 \\
\pm 1,05 \\
\end{array}$ & $\begin{array}{l}6,58 \pm \\
0,91 \\
\end{array}$ & $\begin{array}{l}1 \\
213,00 \pm 19\end{array}$ & $\begin{array}{l}607,00 \pm 8, \\
49\end{array}$ & $\begin{array}{l}6,23 \pm 1, \\
82\end{array}$ & $\begin{array}{l}3,80 \pm \\
0,28 \\
\end{array}$ & $\begin{array}{l}21,12 \pm \\
4,98 \\
\end{array}$ & $\begin{array}{l}216,00 \pm 5, \\
66\end{array}$ & $\begin{array}{l}1,80 \pm 0 \\
00\end{array}$ & $\begin{array}{l}1,39 \pm \\
0,00 \\
\end{array}$ & $\begin{array}{l}1,31 \pm 1, \\
28\end{array}$ \\
\hline
\end{tabular}




\begin{tabular}{|c|c|c|c|c|c|c|c|c|c|c|c|c|c|c|c|}
\hline & & & & & & & 80 & & & & & & & & \\
\hline \multirow[b]{2}{*}{ MlP } & PSP & $\begin{array}{l}25,88 \\
\pm 1,03\end{array}$ & $\begin{array}{l}17,00 \pm 1 \\
5,56\end{array}$ & $\begin{array}{l}33,00 \\
\pm 39,60\end{array}$ & $\begin{array}{l}9,00 \\
\pm 10,04\end{array}$ & $\begin{array}{l}5,83 \pm \\
0,69\end{array}$ & $\begin{array}{l}635,75 \pm 32 \\
9,56\end{array}$ & $\begin{array}{l}318,25 \pm 1 \\
65,20\end{array}$ & $\begin{array}{l}4,84 \pm 1, \\
57\end{array}$ & $\begin{array}{l}5,63 \pm \\
2,21\end{array}$ & $\begin{array}{l}25,08 \pm \\
2,64\end{array}$ & $\begin{array}{l}84,50 \pm 11 \\
7,92\end{array}$ & $\begin{array}{l}7,15 \pm 9, \\
12\end{array}$ & $\begin{array}{l}1,04 \pm \\
1,18\end{array}$ & $\begin{array}{l}8,28 \pm 5, \\
08\end{array}$ \\
\hline & PSS & $\begin{array}{l}24,00 \\
\pm 0,00 \\
\end{array}$ & $\begin{array}{l}8,75 \pm 9,6 \\
0\end{array}$ & $\begin{array}{l}15,00 \pm 14 \\
, 12\end{array}$ & $\begin{array}{l}3,40 \pm 2,0 \\
0\end{array}$ & $\begin{array}{l}5,78 \pm \\
0,07 \\
\end{array}$ & \begin{tabular}{|l}
$470,50 \pm 0,7$ \\
1 \\
\end{tabular} & $\begin{array}{l}235,00 \pm 0, \\
00\end{array}$ & $\begin{array}{l}2,47 \pm 1, \\
26\end{array}$ & $\begin{array}{l}3,70 \pm \\
0,14 \\
\end{array}$ & $\begin{array}{l}23,76 \pm \\
1,24 \\
\end{array}$ & $\begin{array}{l}24,00 \pm 16, \\
97\end{array}$ & $\begin{array}{l}6,40 \pm 0, \\
00\end{array}$ & $\begin{array}{l}1,36 \pm \\
0,00 \\
\end{array}$ & $\begin{array}{l}6,75 \pm 8, \\
70\end{array}$ \\
\hline \multirow[b]{2}{*}{ EtS } & PSP & $\begin{array}{l}24,25 \\
\pm 0,29\end{array}$ & $\begin{array}{l}11,25 \pm 2, \\
63\end{array}$ & $\begin{array}{l}14,00 \\
\pm 8,83\end{array}$ & $\begin{array}{l}8,25 \pm 8,7 \\
7\end{array}$ & $\begin{array}{l}5,31 \pm \\
0,99\end{array}$ & $\begin{array}{l}216,50 \pm 16 \\
60\end{array}$ & $\begin{array}{l}108,25 \pm 7, \\
93\end{array}$ & $\begin{array}{l}6,36 \pm 2, \\
84\end{array}$ & $\begin{array}{l}6,13 \pm \\
1,61\end{array}$ & $\begin{array}{l}23,32 \pm \\
5,82\end{array}$ & $\begin{array}{l}22,50 \pm 21, \\
81\end{array}$ & $\begin{array}{l}3,50 \pm 2, \\
40\end{array}$ & $\begin{array}{l}1,91 \pm \\
0,45\end{array}$ & $\begin{array}{l}1,44 \pm 0, \\
41\end{array}$ \\
\hline & PSS & $\begin{array}{l}24,25 \\
\pm 0,35 \\
\end{array}$ & $\begin{array}{r}12,50 \\
\pm 7,78 \\
\end{array}$ & $\begin{array}{l}17,00 \pm 1, \\
41\end{array}$ & $\begin{array}{l}10,10 \pm 4, \\
10\end{array}$ & $\begin{array}{l}5,54 \pm \\
0,30 \\
\end{array}$ & $\begin{array}{l}205,50 \pm 89, \\
80\end{array}$ & $\begin{array}{l}103,00 \pm 4 \\
5,25\end{array}$ & $\begin{array}{l}2,28 \pm 0, \\
70\end{array}$ & $\begin{array}{l}4,10 \pm \\
0,57 \\
\end{array}$ & $\begin{array}{l}19,36 \pm \\
4,98 \\
\end{array}$ & $\begin{array}{l}18,00 \pm 16, \\
97\end{array}$ & $\begin{array}{l}6,70 \pm 0, \\
00\end{array}$ & $\begin{array}{l}0,27 \pm \\
0,00 \\
\end{array}$ & $\begin{array}{l}0,56 \pm 0, \\
22\end{array}$ \\
\hline \multirow[b]{2}{*}{ EtP } & PSP & $\begin{array}{l}24,75 \pm 0, \\
50\end{array}$ & $\begin{array}{l}42,75 \pm 1 \\
7,21 \\
\end{array}$ & $\begin{array}{l}135,25 \pm 6 \\
3,66 \\
\end{array}$ & $\begin{array}{l}19,09 \pm 1 \\
3,03\end{array}$ & $\begin{array}{l}6,57 \pm \\
0,13 \\
\end{array}$ & $\begin{array}{l}196,75 \pm 23, \\
03\end{array}$ & $\begin{array}{l}98,25 \pm 11, \\
59\end{array}$ & $\begin{array}{l}6,36 \pm 2, \\
84\end{array}$ & $\begin{array}{l}6,48 \pm \\
3,77 \\
\end{array}$ & $\begin{array}{l}21,56 \pm \\
3,01 \\
\end{array}$ & $\begin{array}{l}32,50 \pm 9,7 \\
1 \\
\end{array}$ & $\begin{array}{l}6,40 \pm 6, \\
79\end{array}$ & $\begin{array}{l}1,59 \pm \\
0,00 \\
\end{array}$ & $\begin{array}{l}22,53 \pm \\
4,41 \\
\end{array}$ \\
\hline & PSS & $\begin{array}{l}23,50 \pm 0, \\
71\end{array}$ & $\begin{array}{l}13,50 \\
\pm 6,36\end{array}$ & $\begin{array}{l}29,50 \pm 13 \\
, 44\end{array}$ & $\begin{array}{l}17,78 \pm 1 \\
3,05\end{array}$ & $\begin{array}{l}6,65 \pm \\
0,15\end{array}$ & $\begin{array}{l}178,00 \pm 2,8 \\
3\end{array}$ & $\begin{array}{l}89,00 \pm 1,4 \\
1\end{array}$ & $\begin{array}{l}2,28 \pm 0, \\
70\end{array}$ & $\begin{array}{l}3,25 \pm \\
0,07\end{array}$ & $\begin{array}{l}19,26 \pm \\
5,12\end{array}$ & $\begin{array}{l}26,00 \pm 5,6 \\
6\end{array}$ & $\begin{array}{l}2,60 \pm 0, \\
00\end{array}$ & $\begin{array}{l}0,65 \pm \\
0,00\end{array}$ & $\begin{array}{l}15,60 \pm \\
1,57\end{array}$ \\
\hline \multirow[b]{2}{*}{ ObS } & PSP & $\begin{array}{l}26,13 \pm 1, \\
31\end{array}$ & $\begin{array}{l}9,00 \\
\pm 10,80\end{array}$ & $\begin{array}{l}6,75 \\
\pm 7,09\end{array}$ & $\begin{array}{l}2,73 \\
\pm 2,32\end{array}$ & $\begin{array}{l}5,41 \pm \\
0,08\end{array}$ & $\begin{array}{l}148,25 \pm 6,0 \\
8\end{array}$ & $\begin{array}{l}74,00 \pm 3,4 \\
6\end{array}$ & $\begin{array}{l}5,43 \pm 2, \\
18\end{array}$ & $\begin{array}{l}5,53 \pm \\
2,59\end{array}$ & $\begin{array}{l}27,28 \pm \\
1,76\end{array}$ & $\begin{array}{l}45,50 \pm 51, \\
65\end{array}$ & $\begin{array}{l}5,10 \pm 6, \\
79\end{array}$ & $\begin{array}{l}2,55 \pm \\
3,27\end{array}$ & $\begin{array}{l}1,87 \pm 0, \\
44\end{array}$ \\
\hline & PSS & $\begin{array}{l}25,00 \\
\pm 0,71 \\
\end{array}$ & $\begin{array}{l}6,50 \pm 0,7 \\
1\end{array}$ & $\begin{array}{l}9,50 \\
\pm 0,71 \\
\end{array}$ & $\begin{array}{l}4,50 \pm 0,7 \\
1\end{array}$ & $\begin{array}{l}5,66 \pm \\
0,11\end{array}$ & $\begin{array}{l}144,00 \pm 2,8 \\
3\end{array}$ & $\begin{array}{l}72,00 \pm 1,4 \\
1\end{array}$ & $\begin{array}{l}1,19 \pm 1, \\
39\end{array}$ & $\begin{array}{l}4,15 \pm \\
0,49\end{array}$ & $\begin{array}{l}11,44 \pm \\
3,73 \\
\end{array}$ & $\begin{array}{l}27,00 \pm 32, \\
53\end{array}$ & $\begin{array}{l}7,30 \pm 0, \\
00\end{array}$ & $\begin{array}{l}0,33 \pm \\
0,00\end{array}$ & $\begin{array}{l}1,97 \pm 0, \\
06\end{array}$ \\
\hline \multirow[b]{2}{*}{ ObP } & PSP & $\begin{array}{l}26,25 \pm 0, \\
65\end{array}$ & $\begin{array}{l}17,75 \\
\pm 13,89 \\
\end{array}$ & $\begin{array}{l}20,75 \pm 10 \\
, 90\end{array}$ & $\begin{array}{l}8,03 \\
\pm 5,42 \\
\end{array}$ & $\begin{array}{l}5,92 \pm \\
1,02 \\
\end{array}$ & $\begin{array}{l}144,00 \pm 9,8 \\
3 \\
\end{array}$ & $\begin{array}{l}72,00 \pm 4,5 \\
5\end{array}$ & $\begin{array}{l}4,74 \pm 2, \\
27\end{array}$ & $\begin{array}{l}6,00 \pm \\
2,20 \\
\end{array}$ & $\begin{array}{l}17,60 \pm \\
8,01 \\
\end{array}$ & $\begin{array}{l}43,00 \pm 37, \\
26\end{array}$ & $\begin{array}{l}0,75 \pm 0, \\
92\end{array}$ & $\begin{array}{l}0,86 \pm \\
0,95 \\
\end{array}$ & $\begin{array}{l}25,82 \pm \\
1,93 \\
\end{array}$ \\
\hline & PSS & $\begin{array}{l}25,50 \pm 0, \\
71\end{array}$ & $\begin{array}{l}4,00 \\
\pm 1,41 \\
\end{array}$ & $\begin{array}{l}4,00 \pm 2,8 \\
3\end{array}$ & $\begin{array}{l}1,50 \pm 0,7 \\
1\end{array}$ & $\begin{array}{l}6,02 \pm \\
0,88 \\
\end{array}$ & $\begin{array}{l}135,00 \pm 0,0 \\
0\end{array}$ & \begin{tabular}{|l|}
$67,00 \pm 0,0$ \\
0 \\
\end{tabular} & $\begin{array}{l}1,88 \pm 0, \\
70\end{array}$ & $\begin{array}{l}4,10 \pm \\
0,14 \\
\end{array}$ & $\begin{array}{l}13,20 \pm \\
1,24 \\
\end{array}$ & $\begin{array}{l}46,00 \pm 50, \\
91\end{array}$ & $\begin{array}{l}1,90 \pm 0, \\
00\end{array}$ & $\begin{array}{l}0,03 \pm \\
0,00 \\
\end{array}$ & $\begin{array}{l}13,30 \pm \\
4,38 \\
\end{array}$ \\
\hline
\end{tabular}

TableauVII: Densités des kystes de différentes espèces de flagellés identifiés dans les sources et puits pendant la période d'étude (en kystes/L)

MES : Matières En Suspension; pH : Potentiel Hydrogène ; TDS : Solides Totaux Dissous; $\mathrm{CO}_{2}$ : Gaz Carbonique ; $\mathrm{O}_{2}$ : Oxygène Dissous ; $\mathrm{NO}_{3}{ }^{-}$: Nitrate $; \mathrm{PO}_{4}{ }^{3}$ : Orthophosphates ; PSP : Petite Saison de pluies; PSS : Petite Saison Sèche 